\title{
Left atrial appendage orifice area and morphology is closely associated with flow velocity in patients with nonvalvular atrial fibrillation
}

\author{
Lei Chen ${ }^{1+}$, Changjiang $\mathrm{Xu}^{2 \dagger}$, Wensu Chen ${ }^{1}$ and Chaoqun Zhang ${ }^{1 *}$
}

\begin{abstract}
Background: Thromboembolic events are the most serious complication of atrial fibrillation (AF), and the left atrial appendage (LAA) is the most important site of thrombosis in patients with AF. During the period of COVID-19, a noninvasive left atrial appendage detection method is particularly important in order to reduce the exposure of the virus. This study used CT three-dimensional reconstruction methods to explore the relationship between LAA morphology, LAA orifice area and its mechanical function in patients with non-valvular atrial fibrillation (NVAF).
\end{abstract}

Methods: A total of 81 consecutive patients with NVAF (36 cases of paroxysmal atrial fibrillation and 45 cases of persistent atrial fibrillation) who were planned to undergo catheter radiofrequency ablation were enrolled. All patients were examined by transthoracic echocardiography (TTE), TEE, and computed tomography angiography (CTA) before surgery. The LAA orifice area was obtained according to the images of CTA. According to the left atrial appendage morphology, it was divided into chicken wing type and non-chicken wing type. At the same time, TEE was performed to determine left atrial appendage flow velocity (LAAFV), and the relationship between the left atrial appendage orifice area and LAAFV was analyzed.

Results: The LAAFV in Non-chicken wing group was lower than that in Chicken wing group $(36.2 \pm 15.0 \mathrm{~cm} / \mathrm{s}$ vs. $49.1 \pm 22.0 \mathrm{~cm} / \mathrm{s}, p$-value $<0.05$ ). In the subgroup analysis, the LAAFV in Non-chicken wing group was lower than that in Chicken wing group in the paroxysmal AF $(44.0 \pm 14.3 \mathrm{~cm} / \mathrm{s}$ vs. $60.2 \pm 22.8 \mathrm{~cm} / \mathrm{s}, p$-value $<0.05)$. In the persistent $A F$, similar results were observed $(29.7 \pm 12.4 \mathrm{~cm} / \mathrm{s}$ vs. $40.8 \pm 17.7 \mathrm{~cm} / \mathrm{s}, p$-value $<0.05)$. The LAAFV in persistent AF group was lower than that in paroxysmal AF group $(34.6 \pm 15.8 \mathrm{~cm} / \mathrm{s}$ vs. $49.9 \pm 20.0 \mathrm{~cm} / \mathrm{s}, p$-value $<0.001)$. The LAAFV was negatively correlated with left atrial dimension $(R=-0.451, p$-value $<0.001)$, LAA orifice area $(R=-0.438$, $p$-value $<0.001)$ and left ventricular mass index (LVMI) $(R=-0.624, p$-value $<0.001)$, while it was positively correlated with $\operatorname{LVEF}(R=0.271, p$-value $=0.014)$. Multiple linear regression analysis showed that LAA morphology $(\beta=-0.335$, $p$-value $<0.001)$, LAA orifice area $(\beta=-0.185, p$-value $=0.033)$, AF type $(\beta=-0.167, p$-value $=0.043)$ and LVMl $(\beta=-0.465, p$-value $<0.001)$ were independent factors of LAAFV.

*Correspondence: chaoqun1973@sina.com

${ }^{\dagger}$ Lei Chen and Changjiang Xu have contributed equally to this work

${ }^{1}$ Department of Cardiology, The Affiliated Hospital of Xuzhou Medical

University, Xuzhou 221002, Jiangsu, China

Full list of author information is available at the end of the article

(c) The Author(s) 2021. Open Access This article is licensed under a Creative Commons Attribution 4.0 International License, which permits use, sharing, adaptation, distribution and reproduction in any medium or format, as long as you give appropriate credit to the original author(s) and the source, provide a link to the Creative Commons licence, and indicate if changes were made. The images or other third party material in this article are included in the article's Creative Commons licence, unless indicated otherwise in a credit line to the material. If material is not included in the article's Creative Commons licence and your intended use is not permitted by statutory regulation or exceeds the permitted use, you will need to obtain permission directly from the copyright holder. To view a copy of this licence, visit http://creativecommons.org/licenses/by/4.0/. The Creative Commons Public Domain Dedication waiver (http://creativeco mmons.org/publicdomain/zero/1.0/) applies to the data made available in this article, unless otherwise stated in a credit line to the data. 
Conclusions: The LAA orifice area is closely related to the mechanical function of the LAA in patients with NVAF. The larger LAA orifice area and LVMI, Non-chicken wing LAA and persistent AF are independent predictors of decreased mechanical function of LAA, and these parameters might be helpful for better management of LA thrombosis.

Keywords: Atrial fibrillation, Left atrial appendage orifice area, Left atrial appendage mechanical function

\section{Background}

Atrial fibrillation (AF) is currently the most common arrhythmia encountered in clinical practice. Epidemiological surveys show that the incidence of AF in the population is approximately $0.4-1 \%$. With the aging of the global population, the incidence rate is gradually increasing. It is estimated that, by 2035 , the incidence of AF will double the current incidence [1]. AF poses a huge threat and can cause serious damage to the life and health of patients, greatly increasing the risk of ischemic stroke, systemic artery embolism, heart failure, and other diseases, as well as having high disability and fatality rates [2-5]. Thromboembolic events are the most serious complication of AF. Studies have shown that the left atrial appendage is the most important site of thrombosis in patients with $\mathrm{AF}$, and nonvalvular atrial fibrillation (NVAF) thrombi are almost all located in the left atrial appendage [6, 7]. Previous studies have shown that left atrial appendage flow velocity (LAAFV) is closely related to the formation of left atrial mural thrombus and spontaneous imaging [8]. Transesophageal echocardiography (TEE) is currently the most widely used examination method to assess left atrial appendage function and thrombosis [9], but this examination is semi-invasive. The examination process is relatively painful, and there are many contraindications. In addition, the coronavirus disease-19 (COVID-19) has spread globally and is still difficult to control, during the period of COVID-19, the guidelines recommend that it is best to use noninvasive imaging methods to reduce the risk of virus exposure [10-12]. With the development of CT and $3 \mathrm{D}$ reconstruction technologies, 3D CT reconstruction has become a simple and reliable way to understand the structure and morphology of the left atrial appendage $[7,8]$. At present, there are few relevant studies on the left atrial appendage (LAA) morphology or orifice area on its mechanical function, and there are still controversies. This study investigates the relationship between LAA morphology, LAA orifice area and its mechanical function in patients with NVAF and attempts to identify an effective factor that can predict the reduction in mechanical function of LAA.

\section{Methods}

\section{Research objects}

A retrospective analysis of patients with NVAF who were hospitalized in the Department of Cardiology, Affiliated Hospital of Xuzhou Medical University from November 2016 to November 2020. All patients received standardized drug treatment and management. Inclusion criteria included AF was diagnosed by ECG or 24-h Holter, all patients received TTE, TEE, and CTA examinations, and the interval between TEE and CTA examinations was within $48 \mathrm{~h}$, patients with paroxysmal AF (PaAF) show sinus rhythm during TEE examination, and those with persistent AF (PeAF) show an AF rhythm. Exclusion criteria included incomplete clinical data, complications with valvular heart disease (moderate to severe mitral valve stenosis), patent foramen ovale, artificial valve replacement, poor CT image quality and complete LAA data that could not be obtained, severe liver and kidney dysfunction, patients with thyroid disease or multiple organ dysfunction, etc. Finally, a total of 81 patients were selected, including 36 cases (44.4\%) with PaAF and 45 cases (55.6\%) with PeAF, with an average age of $61.8 \pm 8.8$ years. The basic clinical data of the patients are shown in Table 1.

\section{Diagnostic criteria for atrial fibrillation}

The electrocardiogram or 24-h Holter electrocardiogram showed the disappearance of $\mathrm{P}$ waves and replaced them with $f$ waves of different sizes, shapes, and amplitudes. The frequency of the $\mathrm{f}$ wave is 350 to 600 times/min, and the R-R interval is absolutely unequal (Fig. 1). According to the duration of AF, it is divided into PaAF and PeAF. $\mathrm{PaAF}$ is defined as AF that terminates within 7 days, and PeAF is defined as AF that lasts more than 7 days [13].

\section{Transthoracic echocardiography (TTE) examination}

A Philips EPIQ 7c ultrasonic diagnostic apparatus, S5-1 probe, and probe with a frequency $1-5 \mathrm{MHz}$ were used. The patient was placed in the left decubitus position, and the patient's left atrium anteroposterior diameter, left ventricular end-diastolic diameter, left ventricular ejection fraction (measured by biplane 
Table 1 Clinical data of the study population

\begin{tabular}{lllll}
\hline Variables & All population $(\boldsymbol{n}=\mathbf{8 1})$ & PaAF $(\boldsymbol{n}=\mathbf{3 6})$ & PeAF $(\boldsymbol{n}=\mathbf{4 5})$ & $\boldsymbol{P}$-value \\
\hline Age (years) & $61.8 \pm 8.8$ & $63.2 \pm 8.8$ & $60.7 \pm 8.8$ & 0.198 \\
Male $(n, \%)$ & $57(70.4)$ & $26(72.2)$ & $31(68.9)$ & 0.744 \\
BMI $\left(\mathrm{kg} / \mathrm{m}^{2}\right)$ & $25.6 \pm 3.0$ & $25.5 \pm 3.1$ & $25.7 \pm 3.0$ & 0.722 \\
Hypertension $(n, \%)$ & $30(37.0)$ & $12(33.3)$ & $18(40.0)$ & 0.537 \\
Diabetes $(n, \%)$ & $11(13.6)$ & $5(13.9)$ & $6(13.3)$ & 1.000 \\
CAD $(n, \%)$ & $27(33.3)$ & $13(36.1)$ & $14(31.1)$ & 0.635 \\
Past Stroke $(n, \%)$ & $16(19.8)$ & $5(13.9)$ & $5(24.4)$ & 0.236 \\
CHF $(n, \%)$ & $11(13.6)$ & $6(16.7)$ & $11(24)$ & 0.690 \\
Smokers $(n, \%)$ & $24(29.6)$ & $13(36.1)$ & $2.1)$ & 0.253 \\
$\mathrm{CHA}_{2} \mathrm{DS}_{2}$-VAScscores & $2.1 \pm 1.6$ & $2.1 \pm 1.6$ & & 0.730 \\
\hline
\end{tabular}

$B M I$ Body mass index, $C A D$ coronary artery disease, $C H F$ congestive heart failure

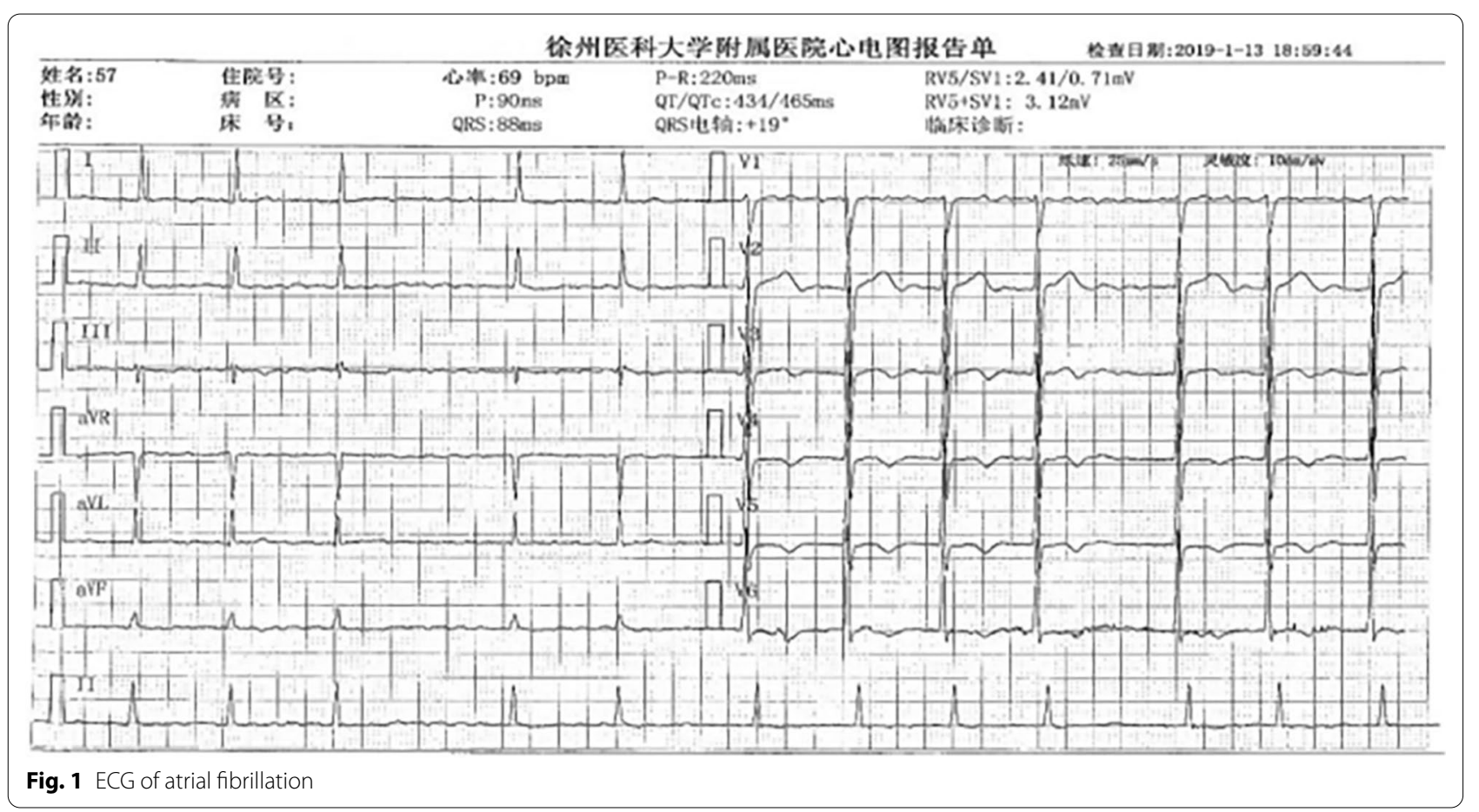

Simpson method), left ventricular posterior wall thickness, ventricular septal thickness and other parameters were recorded in detail. The Devereux formula [14] was used to calculate the left ventricular mass index (LVMI), as follows: LVMI $\left(\mathrm{g} / \mathrm{m}^{2}\right)=\mathrm{LVM}$ (calculated by $0.8 \times 1.04$ $\left.\left[(\mathrm{IVSd}+\mathrm{PWTd}+\mathrm{LVDd})^{3}-\mathrm{LVDd}^{3}\right]+0.6\right) /$ BSA (calculated by $0.006 \times$ height $(\mathrm{cm})+0.013 \times$ body weight $(\mathrm{kg})$ $-0.153)$.

\section{TEE examination and the determination of LAAFV}

A Philips iE33 color Doppler ultrasound diagnostic apparatus and a $\mathrm{X} 7-2 \mathrm{t}$ transesophageal matrix real-time $3 \mathrm{D}$ probe with a frequency of $2-7 \mathrm{MHz}$ were used. Before the examination, patients fasted for 6-8 h. They were connected to the ECG synchronization recording, the pulsed wave Doppler sampling volume was placed within one-third of the proximal opening of the LAA, and the LAA blood flow spectrum was obtained. The blood flow spectrum of the LAA is a regular two-way wave in sinus rhythm and an irregular sawtooth waveform in AF. Record the peak value of the positive wave (maximum emptying speed of the left atrial appendage) within 3 cardiac cycles, and take the average value as left atrial appendage flow velocity (LAAFV) [15] (Figs. 2 and 3). 


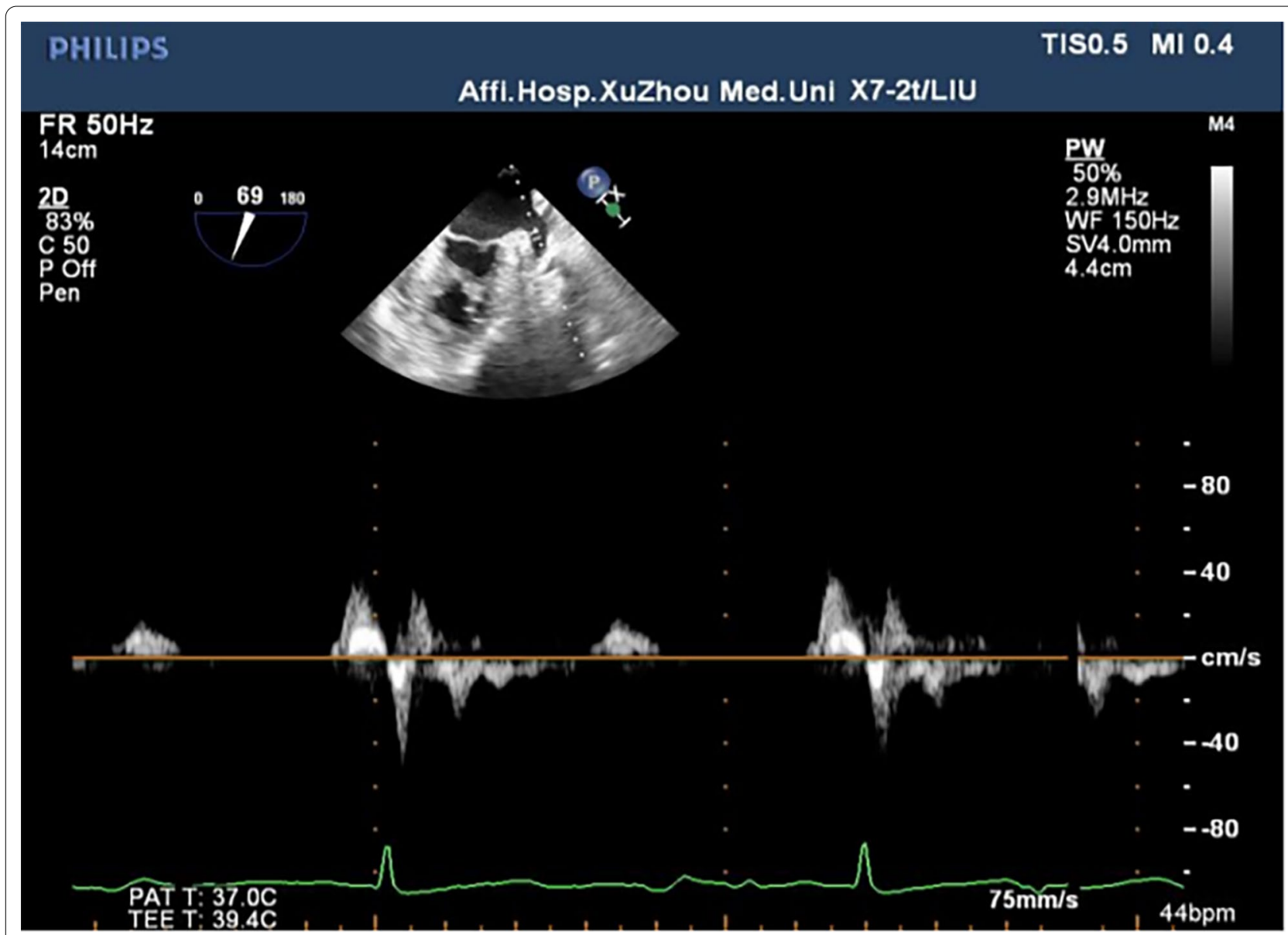

Fig. 2 The blood flow pattern of the left atrial appendage in sinus rhythm

\section{Computed tomography angiography (CTA) and 3D reconstruction}

The CT imaging data were acquired by the German Siemens dual-source CT machine (SOMATOM Definition, SIEMENS Germany). Iodomidol (60-80 $\mathrm{ml})$ was injected into the cubital vein at a flow rate of 5 $\mathrm{ml} / \mathrm{s}$, and then $50 \mathrm{ml}$ of normal saline was injected at a rate of $5 \mathrm{ml} / \mathrm{s}$. Contrast agent tracking technology triggered enhanced scanning. The following scan parameters were used-trigger plane: ascending aorta root level, trigger threshold: $90-100 \mathrm{Hu}$, start scanning after $6 \mathrm{~s}$ delay, scanning time $5-12 \mathrm{~s}$, scanning range: $1 \mathrm{~cm}$ below tracheal carina to $1.5 \mathrm{~cm}$ lower edge of the heart, detector width $2.0 \mathrm{~mm} \times 32.0 \mathrm{~mm} \times 0.6 \mathrm{~mm}$, layer thickness $2.0 \mathrm{~mm} \times 64.0 \mathrm{~mm} \times 0.6 \mathrm{~mm}$, frame rotation time $330 \mathrm{~ms}$, heart rate dependent pitch $0.2-$ 0.5 , tube current $400 \mathrm{~mA}$, and voltage $120 \mathrm{kV}$.

\section{Measurement of LAA volume and its morphology}

The GE AW4.6 Workstation was used to perform 3D reconstruction of the original $\mathrm{CT}$ images to obtain $3 \mathrm{D}$ images of the LAA and left atrium (LA). Then, a cutting tool was used to separate the LAA from the LA to obtain the LAA volume. According to the morphological characteristics of the LAA, it can be divided into chicken wing (there is an obvious fold at the proximal or middle part of the main lobe of the left atrial appendage) and non-chicken wing (other forms beside chicken wings) (Figs. 4 and 5).

\section{Measurement of the LAA orifice area}

The long diameter (D1) and short diameter (D2) of the LAA orifice were measured by a Philips IntelliSpace Portal workstation. The LAA orifice was manually cross-sectioned from the multiplanar reconstruction image, and the orifice area was determined by its narrowest part. By creating a plane perpendicular to the axis of the left atrial ear neck, a cross-sectional view of the LAA is generated 


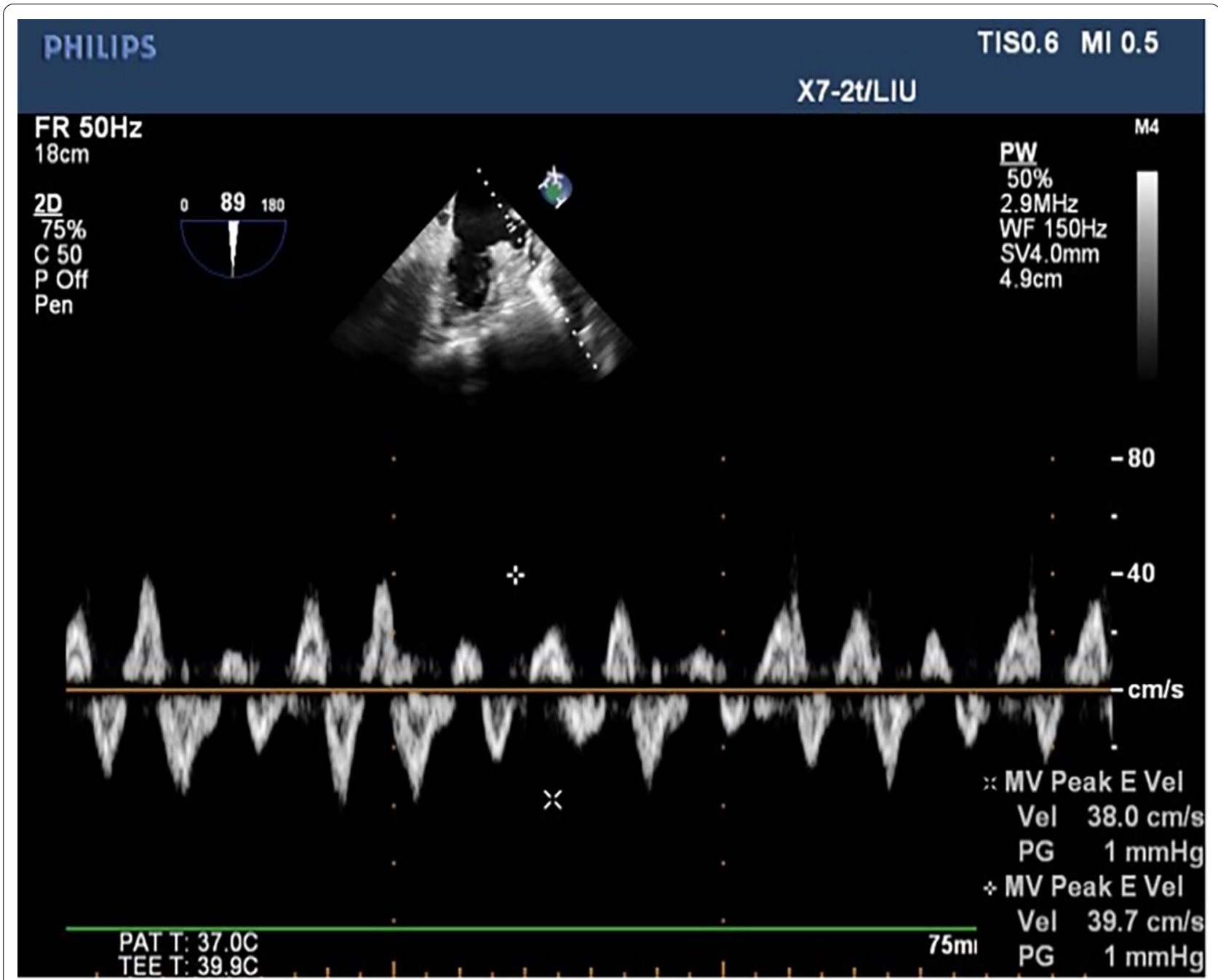

Fig. 3 The blood flow pattern of the left atrial appendage in AF rhythm

(Fig. 6). The formula $0.785^{*} \mathrm{D} 1 * \mathrm{D} 2$ was used to calculate the LAA orifice area [16].

\section{Statistical analysis}

SPSS 22.0 software was used for statistical analysis. Measurement data are expressed as the mean \pm standard deviation, and independent sample t-test was used for comparison between groups. Count data are expressed by the number of cases and percentage (\%), and the $x^{2}$ test or Fisher's exact probability method was used for comparison between groups. T-test or simple linear regression analysis was used to determine each parameter that may affect LAAFV. Then, the findings were incorporated into multiple linear regression analysis to evaluate the independent predictors that determine LAAFV. $P$-value $<0.05$ was considered statistically significant.

\section{Results}

Comparison of baseline data

As shown in Table 1. There were no significant differences in age, sex, BMI, hypertension, diabetes, coronary artery disease, past stroke, congestive heart failure, smokers and CHA2DS2-VASc scores between PeAF group and PaAF group ( $p$-value $>0.05)$.

\section{Comparison of ultrasound and 3D CT reconstruction of left} atrial appendage data

There were no significant differences in LVEDD, LVST, LVPWT, LVEF, E/e' between PeAF group and PaAF group ( $p$-value $>0.05)$. The LAD and LAA orifice area in PeAF group were larger than that in PaAF group ( $p$-value $<0.05)$, and LAAFV was lower than that in PaAF group $(p$-value $<0.001)$. There was no significant difference in LAA morphology and 

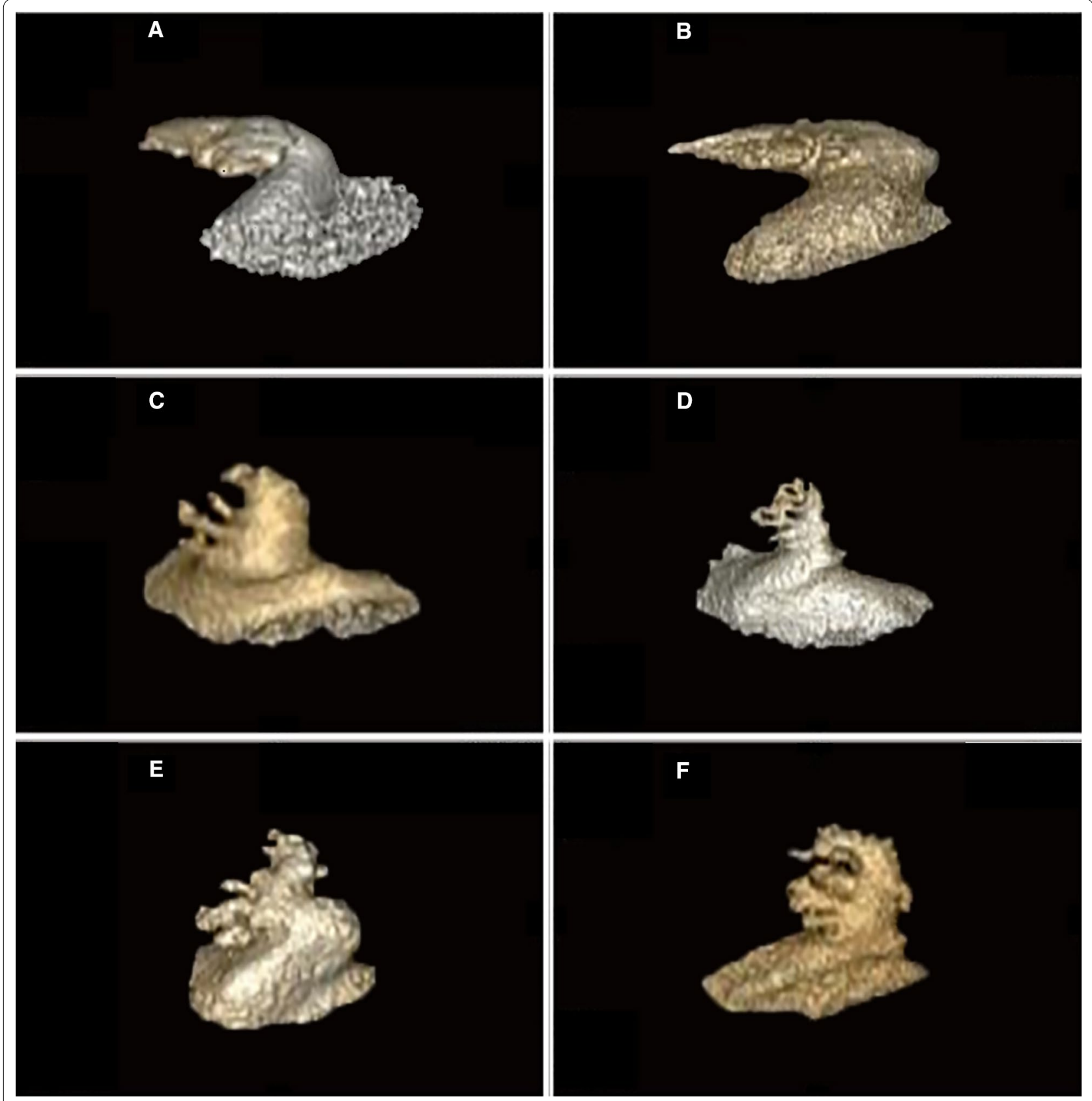

Fig. 4 The shape of the LAA. A, B Chicken-wing. C-F Non-chicken wing

LAA volume between the two groups through CT $(p$-value $>0.05)$. However, the LVMI in PeAF group was larger than that in PaAF group $(p$-value $<0.001)$ (Table 2).

\section{Analysis of relevant parameters of LAAFV by t-test.}

Among all the enrolled patients, the LAAFV in Non-chicken wing group was lower compared with that in Chicken wing group $(36.2 \pm 15.0 \mathrm{~cm} / \mathrm{s}$ vs.
$49.1 \pm 22.0 \mathrm{~cm} / \mathrm{s}, p$-value $<0.05)$ (Fig. 7A). Among different types of AF, the LAAFV in PeAF group was lower compared with that in PaAF group $(34.6 \pm 15.8 \mathrm{~cm} / \mathrm{s}$ vs. $49.9 \pm 20.0 \mathrm{~cm} / \mathrm{s}, p$-value $<0.001$ ) (Fig. 7B). In the subgroup analysis, the LAAFV in Non-chicken wing group was lower than that in Chicken wing group in PaAF $(44.0 \pm 14.3 \mathrm{~cm} / \mathrm{s}$ vs. $60.2 \pm 22.8 \mathrm{~cm} / \mathrm{s}, p$-value $<0.05)$; The LAAFV in Non-chicken wing group was also lower than that in Chicken wing group in PeAF 


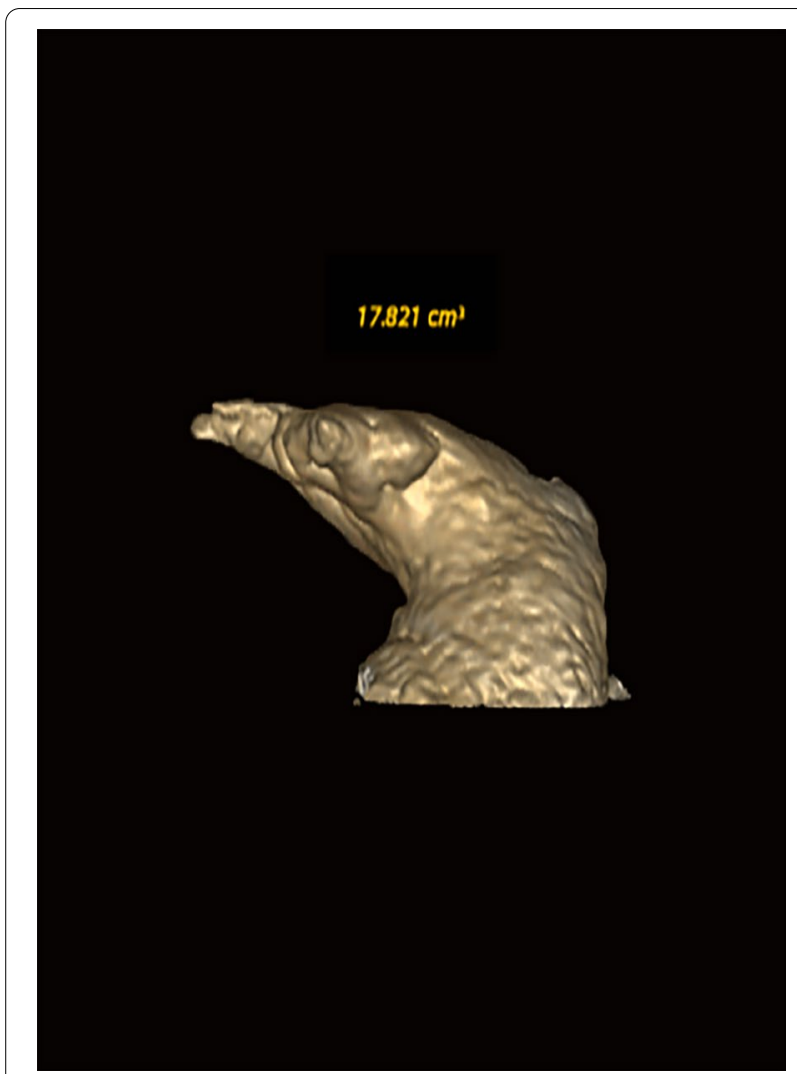

Fig. 5 Left atrial appendage volume

$(29.7 \pm 12.4 \mathrm{~cm} / \mathrm{s}$ vs. $40.8 \pm 17.7 \mathrm{~cm} / \mathrm{s}, p$-value $<0.05)$ (Fig. 7 C).

Analysis of relevant parameters of LAAFV by simple linear regression

The LAAFV was negatively correlated with the LAD $(\mathrm{R}=-0.451, p$-value $<0.001)$ (Fig. $8 \mathrm{~A})$, the LAA orifice area $(\mathrm{R}=-0.438, p$-value $<0.001)$ (Fig. $8 \mathrm{C}$ ) and LVMI $(\mathrm{R}=-0.624, p$-value $<0.001)$ (Fig. $8 \mathrm{D})$. While it was positively correlated with the LVEF $(\mathrm{R}=0.271$, $p$-value $=0.014)($ Fig. 8 B).

\section{Multiple linear regression analysis of LAAFV predictors.}

The statistically significant variables were included in the multiple linear regression analysis. The multiple linear regression analysis showed that LAA morphology $(\beta=-0.335, p$-value $<0.001)$, LAA orifice area $(\beta=-0.185, p$-value $=0.033)$, AF type (paroxysmal vs. persistent $)(\beta=-0.167, p$-value $=0.043)$ and LVMI $(\beta=-0.465, p$-value $<0.001)$ were independent factors of LAAFV (Table 3).

\section{Discussion}

The LAA is a finger-like extension originating from the main body of LA [17]. It has active systolic and diastolic functions, and its mechanical dysfunction may lead to blood flow stagnation and thrombosis [18]. At present, the LAAFV measured by TEE is the most commonly used method to evaluate the mechanical function of the LAA. A large number of studies have shown that the risk of thrombosis is steadily increased with the decrease of LAAFV [19-21]. Handke et al. [8] conducted a TEE study on 500 patients with cerebral ischemia and found that the measurement of the LAAFV may be an important quantitative substitute parameter for evaluating the risk of left atrial thromboembolism. However, TEE is semiinvasive and requires higher personal experience of the operator and may cause complications, such as bleeding and perforation [22]. There are certain checkups, such as combined esophageal stenosis. Therefore, looking for noninvasive examination indicators that can effectively predict the mechanical function of the LAA has important clinical significance. Especially during the period of COVID-19.

At present, research on the anatomy of the LAA is still rare, and there is no uniform standard for the classification of the LAA. With the rapid development of multislice spiral CT and $3 \mathrm{D}$ reconstruction technology, it is possible to observe heart anatomy and vascular structure in detail. According to CT or MRI images of the heart, Wang [23] et al. divided LAA into four categories: chicken wing type, weathercock type, cauliflower type and cactus type. Among them, the chicken wing type is the most common form. However, due to the complex structure of LAA, sometimes it will show different morphological characteristics when viewed from different angles.It has been reported in the literature that this classification method is subjective [24]. Therefore, we refer to the relevant literature to divide LAA morphology into chicken wing type and non-chicken wing type. This classification can significantly reduce subjectivity. Previous studies have shown that LAA morphology is related to the LAAFV $[25,26]$. Fukushima et al. [26] reported that compared with the cactus type and cauliflower type LAA, the chicken-wing type LAA had a significantly higher flow velocity, but there was no statistical difference compared with the weathercock type. However, the study only included patients with PaAF. Our study found that whether it is in PaAF group or PeAF group, the LAA morphology is closely related to its mechanical function. The LAAFV of chicken-wing AF is higher than that of non-chicken wing AF. The possible mechanisms are as follows: First, chicken-wing patients may have greater muscle mass to contract the LAA [27]. Second, high left 


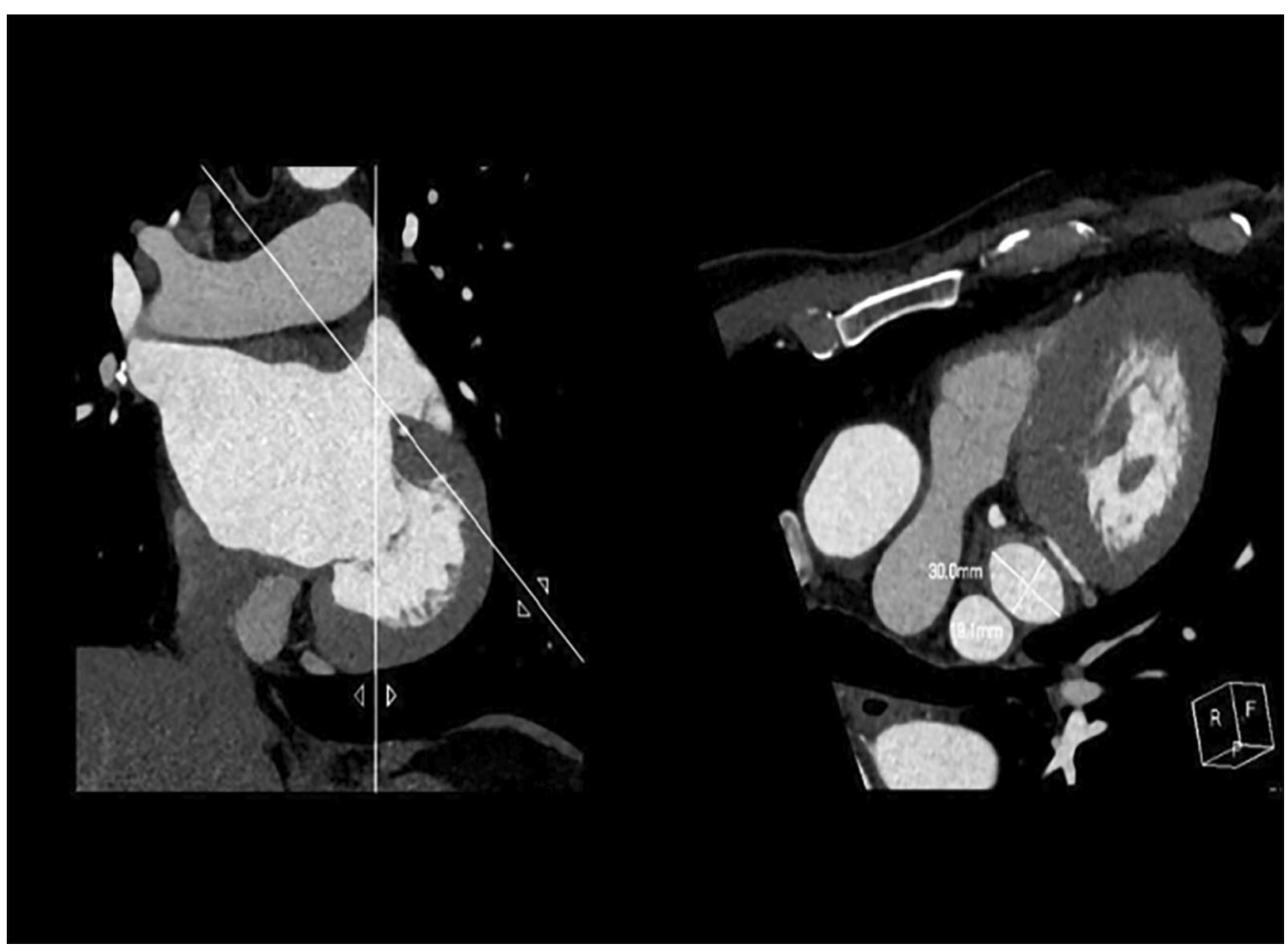

Fig. 6 Measurement of the length and short diameter of the left atrial appendage orifice

Table 2 Ultrasound and 3D CT reconstruction of left atrial appendage data

\begin{tabular}{lllll}
\hline Variables & All population $(\boldsymbol{n}=\mathbf{8 1})$ & PaAF $(\boldsymbol{n}=\mathbf{3 6})$ & PeAF $(\boldsymbol{n}=\mathbf{4 5})$ & $\boldsymbol{P}$-value \\
\hline LAD $(\mathrm{mm})$ & $41.9 \pm 5.3$ & $40.3 \pm 4.3$ & $43.2 \pm 5.7$ & 0.016 \\
LVEDD $(\mathrm{mm})$ & $49.7 \pm 5.2$ & $49.1 \pm 3.5$ & $50.2 \pm 6.2$ & 0.344 \\
IVST $(\mathrm{mm})$ & $9.5 \pm 1.4$ & $9.4 \pm 1.5$ & $9.5 \pm 1.3$ & 0.694 \\
LVPWT $(\mathrm{mm})$ & $9.2 \pm 1.2$ & $9.0 \pm 1.0$ & $9.4 \pm 1.4$ & 0.146 \\
LVEF $(\%)$ & $61.6 \pm 9.8$ & $63.6 \pm 8.4$ & $59.9 \pm 10.7$ & 0.101 \\
E/e' & $8.0 \pm 2.8$ & $7.5 \pm 2.4$ & $8.5 \pm 3.1$ & 0.105 \\
LAAFV $(\mathrm{cm} / \mathrm{s})$ & $41.8 \pm 19.3$ & $50.8 \pm 19.8$ & $34.6 \pm 15.8$ & $<0.001$ \\
Non-chicken wing $(n, \%)$ & $46(56.8)$ & $21(58.3)$ & $25(55.6)$ & 0.802 \\
LAA orifice area $\left(\mathrm{cm}^{2}\right)$ & $4.7 \pm 1.5$ & $4.2 \pm 1.6$ & $5.1 \pm 1.4$ & 0.007 \\
LAA volume $(\mathrm{ml})$ & $11.5 \pm 5.1$ & $10.8 \pm 5.0$ & $12.1 \pm 5.2$ & $111.92 \pm 36.03$ \\
LVMI (g/m $\left.{ }^{2}\right)$ & $101.35 \pm 31.91$ & $88.13 \pm 19.28$ & 0.237 & $<0.001$ \\
\hline
\end{tabular}

LAD left atrial dimension, LVEDD left ventricular end diastolic dimension, IVST interventricular septal thickness, LVPWT left ventricular posterior wall thickness, LVEF left ventricular ejection fraction, LVMI left ventricular mass index

atrial pressure or low left atrial compliance may change LAA morphology[25].

Compared with the direct measurement after CT three-dimensional reconstruction of the LAA, the measurement method used in this study judges that the LAA orifice is relatively less subjective and the measurement repeatability is good [23]. At present, the research on LAA orifice area and the mechanical function of LAA in patients with AF has not been reported in the literature. Our study showed that the increase of LAA orifice area in patients with NVAF is closely related to the decrease of LAAFV. Agmon et al. [28] reported that the emptying speed of the LAA of the normal population was negatively correlated with the diameter of the LAA orifice measured by TEE $(r=-0.29, p$-value $=0.002)$. According to the continuity equation, as the cross-sectional area 
A

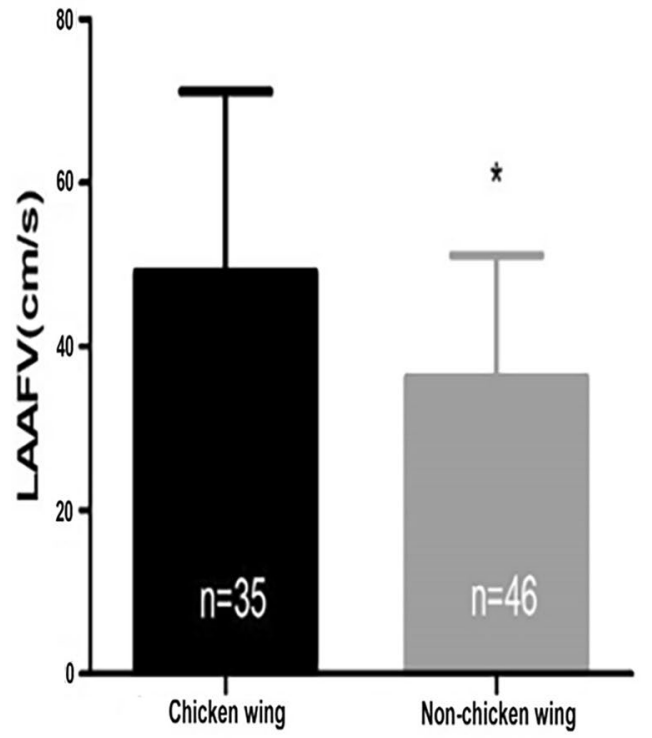

C

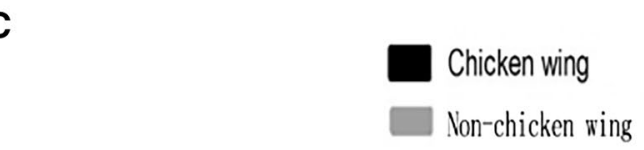

B

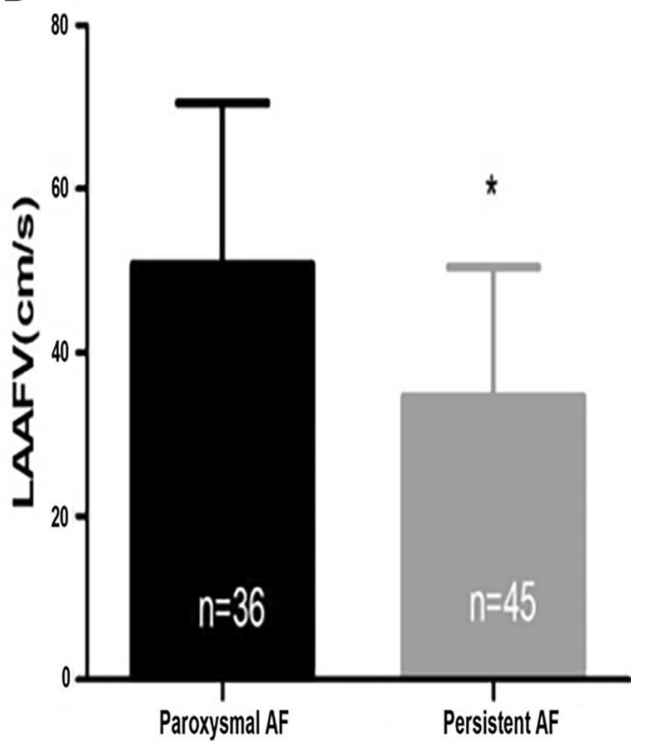

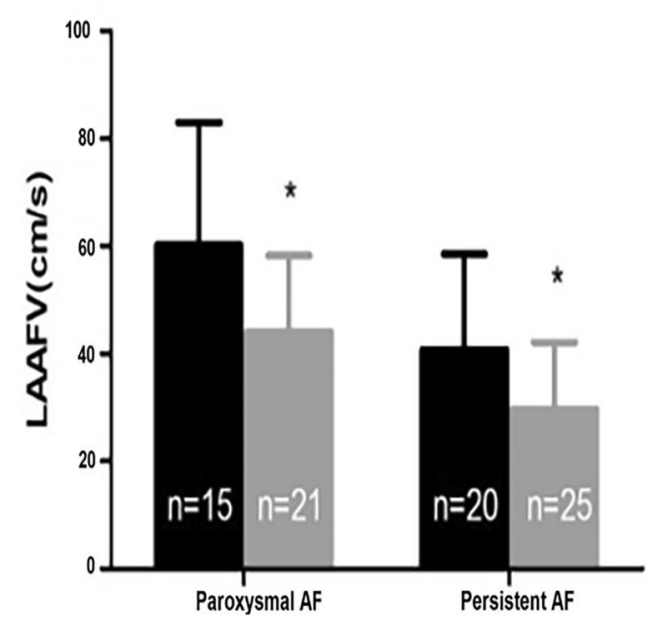

Fig. 7 Analysis of relevant parameters of LAAFV by t test. A Comparison of LAAFV between Chicken wing group and Non-chicken wing group. ${ }^{*} p$-value <0.05: Comparison between Chicken wing and Non-chicken wing. B Comparison of LAAFV between PeAF and PaAF. ${ }^{*} p$-value $<0.05$ : Comparison between PeAF group and PaAF. C Comparison of LAAFV between Chicken wing group and Non-chicken wing group in different types of AF. ${ }^{*} p$-value $<0.05$ : Comparison between Chicken wing and Non-chicken wing

increases, the flow velocity becomes slower. In the case of constant flow, as the cross-sectional area decreases, the flow velocity becomes faster. Our data showed that the relationship between the LAA orifice area and its flow velocity in NVAF patients also conforms to the same law, so LAA orifice area is an important factor in determining LAAFV. Studies have shown that LAAFV was significantly negatively correlated with the LAD [29, 30]. Similar to the results of these studies, we found that LAD is negatively correlated with LAAFV. The reasons may be as follows: With the progress of AF, LA gradually undergoes structural remodeling, which in turn leads to an increase in the inner diameter and pressure of LA. The compliance of LAA is greater than that of the LA and can 

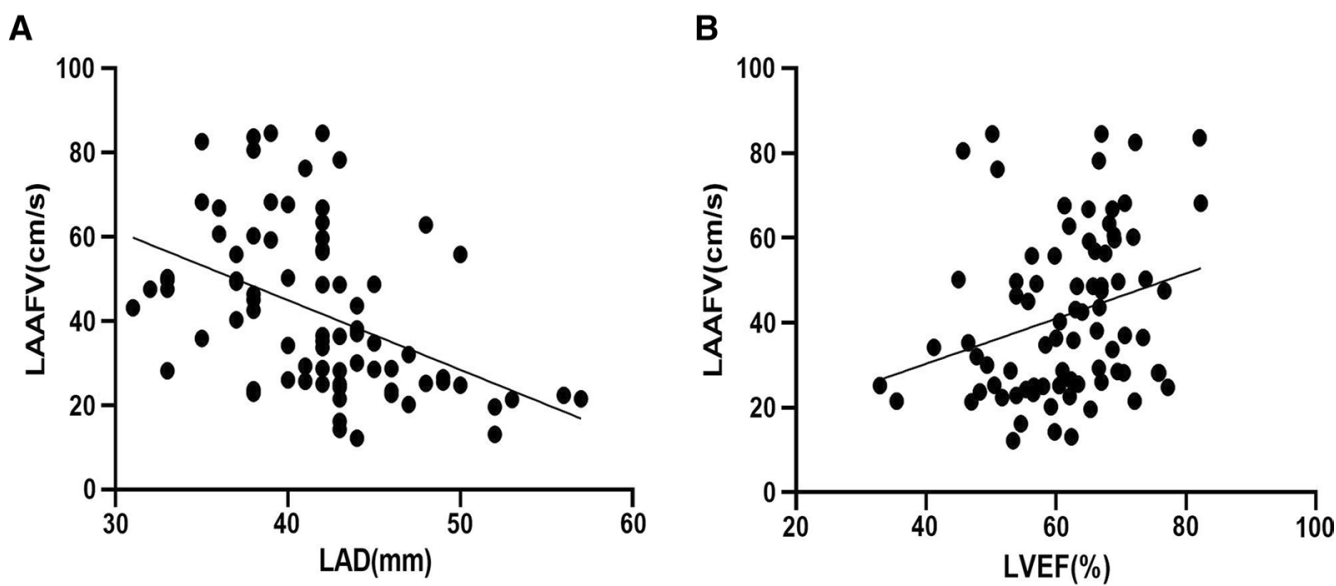

C

D
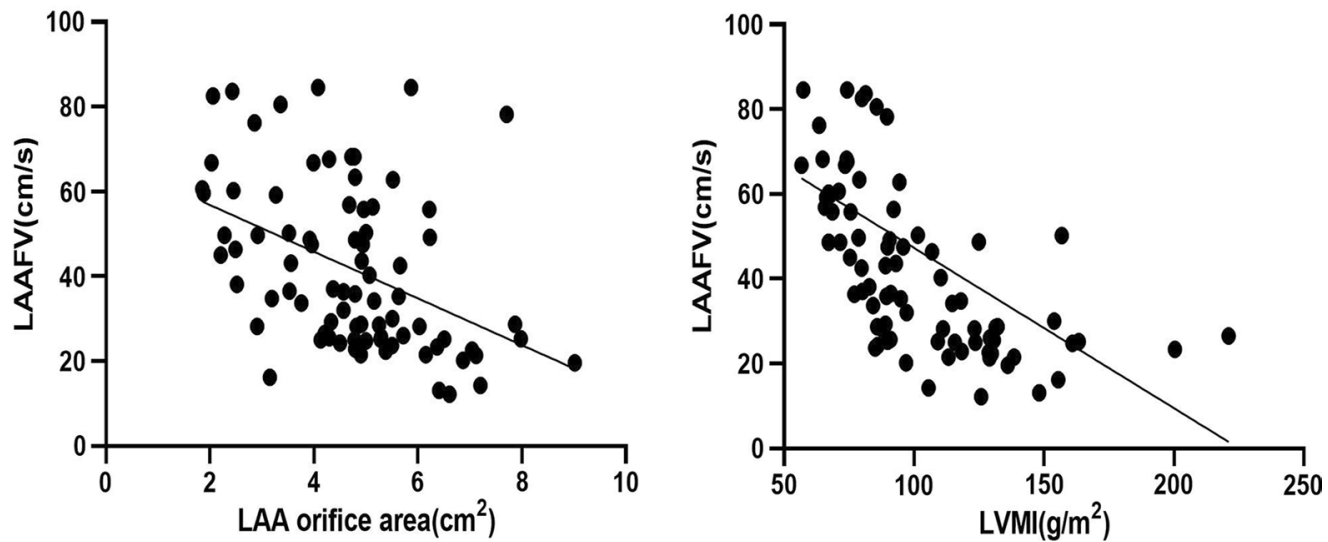

Fig. 8 Analysis of relevant parameters of LAAFV by simple linear regression. A Comparison between LAAFV and LAD $(Y=110.940-1.65 X)$. B Comparison between LAAFV and LVEF $(Y=9.075+0.532 X)$. C Comparison between LAAFV and LAA orifice area $(Y=67.858-5.506 X)$. D Comparison between LAAFV and LVMI $(Y=80.120-0.378 X)$

Table 3 Multiple linear regression analysis of LAAFV predictors

\begin{tabular}{|c|c|c|c|c|c|}
\hline Variables & B & SE & $\beta$ & $T$ & $P$-value \\
\hline Constant & 114.633 & 17.957 & & 6.384 & 0.000 \\
\hline AF type (paroxysmal vs. persistent) & -6.462 & 3.136 & -0.167 & -2.060 & 0.043 \\
\hline LAD & -0.258 & 0.333 & -0.071 & -0.775 & 0.441 \\
\hline LVEF & 0.128 & 0.153 & 0.065 & 0.840 & 0.404 \\
\hline LAA orifice area & -2.324 & 1.068 & -0.185 & -2.177 & 0.033 \\
\hline $\begin{array}{l}\text { LAA morphology (Non-chicken wing vs. } \\
\text { chicken wing) }\end{array}$ & -12.974 & 2.858 & -0.335 & -4.540 & $<0.001$ \\
\hline LVMI & -0.281 & 0.053 & -0.465 & -5.326 & $<0.001$ \\
\hline$R^{2}$ adjusted & 0.569 & & & & \\
\hline$R^{2}$ & 0.602 & & & & \\
\hline
\end{tabular}

LAD left atrial dimension, $L V E F$ left ventricular ejection fraction, $L V M I$ left ventricular mass index

regulate left atrial pressure. However, the increase in left atrial pressure can lead to an increase in the afterload of
LAA and lead to the decrease of LAAFV [31]. This can also explain the view that some scholars believe that LAD can predict the risk of stroke[32]. However, similar to the 
study of Harada $\mathrm{M}$ et al. [33], the multiple linear regression analysis did not prove that LAD is an independent factor of the LAAFV. Therefore, this needs more research to confirm.

The current guidelines recommend that AF be divided into five categories: primary AF, PaAF, PeAF, long-term PeAF and permanent AF [13]. Petersen et al. [34] found that from PaAF group, to PeAF, and then to long-term PeAF group, the LAAFV gradually decreased $(51.4 \pm 25.1 \mathrm{~cm} / \mathrm{s}$ vs. $40.9 \pm 16.3 \mathrm{~cm} / \mathrm{s}$ vs. $29.7 \pm 15.1 \mathrm{~cm} / \mathrm{s}, p$-value $<0.001)$; Multiple linear regression analysis found that AF type was an independent predictor of LAAFV. It is consistent with our research results, and the reasons may be as follows: First, during the TEE and TTE examinations in this study, patients in the PaAF group were required to maintain sinus rhythm. When the heart rhythm is $\mathrm{AF}$, the emptying time of LA and LAA is shortened, so the volume of LA is relatively increased. The rapid and irregular electrical activity will weaken the contractility of LAA, resulting in the decrease of LAAFV. Secondly, PeAF usually has a longer course than PaAF, the electrical remodeling and fibrosis of LA are more serious, which is more likely to cause an increase in the load of the LAA and cause systolic dysfunction, leading to the LAAFV was lower in PeAF group than that in PaAF group. LVEF is a common indicator reflecting left ventricular function. Our study found that LAAFV is positively correlated with LVEF. However, the multiple linear regression analysis did not prove that LVEF is an independent predictor of the LAAFV. This is similar to the study of Kishima et al. [25]. The reason may be that the population included in our study is mainly NVAF patients with normal LVEF. So far, studys on the relationship between LVMI and LAA are rare, and there seems to be some controversy $[33,35]$. Our study showed that LAAFV was negatively correlated with LVMI, and the multiple linear regression analysis proved that LVMI is an independent factor of the LAAFV, which is similar to the study of Harada $M$ et al. [33].

At present, the CHA2DS2-VASc score is the most commonly used index for clinical assessment of stroke risk stratification in NVAF patients, and is used to guide anticoagulation therapy [36]. The 2016 ESC guidelines recommended that patients with CHA2DS2-VASc score $\geq 1$ can choose anticoagulation therapy [37]. However, previous studies have found that patients with a score of 0 are still at risk of ischemic stroke. Gage et al. found that NVAF patients with a CHA2DS2-VASc score of 0 have an annual stroke risk as high as $1.9 \%$ [38]. Moreover, the score mainly focuses on clinical indicators, and does not pay attention to the influence of heart structure and function on thrombosis. Therefore, the scoring system is not sufficient to comprehensively assess the risk of stroke in patients. Our study showed that non-chicken wing LAA in patients with NVAF can cause the decrease of LAAFV, which may increase the risk of thrombosis. Our research on the morphology and mechanical function of LAA may provide additional clinical significance for stroke risk stratification in NVAF patients. However, this study still has some limitations. First, this study is a retrospective, single-center, small-sample study. As a retrospective study, it was regretted that we did not discuss LA volume in this study. It is hoped that there will be a larger cohort for further prospective studies in the future. Second, there is still no uniform standardthe for the LAA morphology. Some clinical studies divide it into four types, this study only classifies it into two types. This classification can minimize the difference between observers, but it may not be precise enough.

\section{Conclusions}

The LAA orifice area is closely related to the mechanical function of the LAA in patients with NVAF. The larger LAA orifice area and LVMI, Non-chicken wing LAA and PeAF are independent factors of decreased mechanical function of LAA, and these parameters might be helpful for better management of LA thrombosis.

\section{Abbreviations}

AF: Atrial fibrillation; BMI: Body mass index; CHF: Congestive heart failure; CTA: Computed tomography angiography; CW: Chicken Wing type; LA: Left atrium; LAA: Left atrial appendage; LAD: Left atrial diameter; LAAFV: Left atrial appendage flow velocity; LVEF: Left ventricular ejection fraction; LVEDD: Left ventricular end diastolic dimension; LVM: Left ventricular mass; LVMI: Left ventricular mass index; IVST: Interventricular septal thickness; LVPWT: Left ventricular posterior wall thickness; TEE: Transesophageal echocardiography; TTE: Transthoracic echocardiography; PaAF: Paroxysmal atrial fibrillation; PeAF: Persistent atrial fibrillation.

\section{Acknowledgements}

Not applicable.

\section{Authors' contributions}

$L C, C X$ and $C Z$ performed the experiments and analyzed the data. $L C$ and $C Z$ designed the study and wrote the manuscript. All authors read and approved the manuscript and agree to be accountable for all aspects of the research in ensuring that the accuracy or integrity of any part of the work are appropriately investigated and resolved. All authors read and approved the final manuscript.

\section{Funding}

Not applicable. 


\section{Availability of data and materials}

The datasets used and/or analyzed during the present study are available from the corresponding author on reasonable request.

\section{Declarations}

\section{Ethics approval and consent to participate}

This study has been approved by the Ethics Committee of the Affiliated Hospital of Xuzhou Medical University (Xuzhou, china), and the ethics number is: XYFY2020-KL178-01. All methods have been implemented in accordance with relevant guidelines and regulations, and all enrolled patients have signed the informed consent.

\section{Consent for publication}

Not applicable.

\section{Competing interests}

The authors declare that they have no competing interests.

\section{Author details}

'Department of Cardiology, The Affiliated Hospital of Xuzhou Medical University, Xuzhou 221002, Jiangsu, China. ${ }^{2}$ Department of Cardiology, The Affiliated Suqian First People's Hospital of Nanjing Medical University, Suqian 223800, Jiangsu, China.

Received: 27 April 2021 Accepted: 1 September 2021

Published online: 16 September 2021

\section{References}

1. Naccarelli GV, et al. Increasing prevalence of atrial fibrillation and flutter in the United States. Am J Cardiol. 2009;104:1534-9.

2. Staerk L, et al. Atrial fibrillation: epidemiology, pathophysiology, and clinical outcomes. Circ Res. 2017;120(9):1501-17.

3. Morin DP, et al. The state of the art: atrial fibrillation epidemiology, prevention, and treatment. Mayo Clinic Proc. 2016;91(12):1778.

4. Soliman EZ, et al. Atrial fibrillation and the risk of myocardial infarction. Jama Internal Med. 2014;174(1):107.

5. Michael, et al. Changes in renal function in patients with atrial fibrillation: an analysis from the RE-LY trial. J Am Coll Cardiol. 2015;65(23):2481-2493.

6. Cresti $A$, et al. Prevalence of extra-appendage thrombosis in nonvalvular atrial fibrillation and atrial flutter in patients undergoing cardioversion: a large transoesophageal echo study. Eurolntervention. 2019;15(3):e225-30.

7. Al-Saady NM, Obel OA, Camm AJ. Left atrial appendage: structure, function, and role in thromboembolism. Heart. 1999;82(5):547-54.

8. Handke $M$, et al. Left atrial appendage flow velocity as a quantitative surrogate parameter for thromboembolic risk: determinants and relationship to spontaneous echocontrast and thrombus formation-a transesophageal echocardiographic study in 500 patients with cerebral ischemia. J Am Soc Echocardiogr. 2005;18(12):1366-72.

9. Saric M, et al. Guidelines for the use of echocardiography in the evaluation of a cardiac source of embolism. J Am Soc Echocardiogr. 2016:29(1):1-42.

10. Skulstad H, et al. COVID-19 pandemic and cardiac imaging: EACVI recommendations on precautions, indications, prioritization, and protection for patients and healthcare personnel. Eur Heart J Cardiovasc Imaging. 2020;21(6):592-8.

11. Kirkpatrick JN, et al. ASE statement on protection of patients and echocardiography service providers during the 2019 novel coronavirus outbreak: endorsed by the American College of Cardiology. J Am Soc Echocardiogr. 2020;33(6):648-53.

12. Choi AD, et al. Society of cardiovascular computed tomography guidance for use of cardiac computed tomography amidst the COVID-19 pandemic endorsed by the American College of Cardiology. J Cardiovasc Comput Tomogr. 2020;14(2):101-4.

13. Hindricks G, et al. ESC Scientific Document Group. 2020 ESC Guidelines for the diagnosis and management of atrial fibrillation developed in collaboration with the European Association for Cardio-Thoracic Surgery (EACTS). Eur Heart J. 2021;42(5):373-498.

14. Jafary FH. Devereux formula for left ventricular mass-be careful to use the right units of measurement. J Am Soc Echocardiogr. 2007;20(6):783-783.

15. Fukushima $K$, et al. Left atrial appendage flow velocity and time from P-wave onset to tissue Doppler-derived A' predict atrial fibrillation recurrence after radiofrequency catheter ablation. Echocardiography. 2015;32(7):1101-8

16. Michel, Haïssaguerre, et al. Spontaneous initiation of atrial fibrillation by ectopic beats originating in the pulmonary veins. N Engl J Med. 1998;339(10):659-66.

17. Beigel $R$, et al. The left atrial appendage: anatomy, function, and noninvasive evaluation. JACC Cardiovasc Imaging. 2014;7(12):1251-65.

18. Kaneko K, et al. Left atrial appendage dysfunction in acute cerebral embolism patients with sinus rhythm: correlation with pulse wave tissue Doppler imaging. Int J Cardiovasc Imaging. 2014;30(7):1245-54.

19. Zabalgoitia M, et al. Transesophageal echocardiographic correlates of clinical risk of thromboembolism in nonvalvular atrial fibrillation. Stroke Prevention in Atrial Fibrillation III Investigators. J Am Coll Cardiol. 1998:31:1622-6.

20. Shinokawa N, et al. A transesophageal echocardiographic study on risk factors for stroke in elderly patients with atrial fibrillation: a comparison with younger patients. Chest. 2001;120:8407-846.

21. Mugge A, et al. Assessment of left atrial appendage function by biplane transesophageal echocardiography in patients with nonrheumatic atrial fibrillation: identification of a subgroup of patients at increased embolic risk. J Am Coll Cardiol. 1994:23:599-607.

22. Daniel W, et al. Safety of transesophageal echocardiography. A multicentre survey of 10419 examinations. Circulation. 1991:83(3):817-21.

23. Wang $Y$, et al. Left atrial appendage studied by computed tomography to help planning for appendage closure device placement. J Cardiovasc Electrophysiol. 2010;21(9):973-82.

24. Di Biase $L$, et al. Left atrial appendage: an underrecognized trigger site of atrial fibrillation. Circulation. 2010;122(2):109-18.

25. Kishima $\mathrm{H}$, et al. Does left atrial appendage morphology influence left atrial appendage flow velocity. Circ J. 2015;79(8):1706-11.

26. Fukushima K, et al. Correlation between left atrial appendage morphology and flow velocity in patients with paroxysmal atrial fibrillation. Eur Heart J Cardiovasc Imaging. 2016;17(1):59-66.

27. Ito T, et al. Influence of altered loading conditions on left atrial appendage function in vivo. Am J Cardiol. 1998;81(8):1056-9.

28. Agmon $Y$, et al. Are left atrial appendage flow velocities adequate surrogates of global left atrial function? A population-based transthoracic and transesophageal echocardiographic study. J Am Soc Echocardiography. 2002;15(5):433-40

29. Watanabe A, et al. Left atrial remodeling assessed by transthoracic echocardiography predicts left atrial appendage flow velocity in patients with paroxysmal atrial fibrillation. Int Heart J. 2016;57(2):177-82.

30. Igarashi Y, et al. Left atrial appendage dysfunction in chronic nonvalvular atrial fibrillation is significantly associated with an elevated level of brain natriuretic peptide and a prothrombotic state. Jpn Circ J. 2001;65(9):788-92.

31. Davis CA Rembert JC, Greenfield JC Compliance of left atrium with and without left atrium appendage. Am J Physiol. 1990;259(4 Pt 2):H1006-8.

32. Hamatani Y, et al. Left atrial enlargement is an independent predictor of stroke and systemic embolism in patients with non-valvular atrial fibrillation. Sci Rep. 2016;6:31042.

33. Harada M, et al. Correlation between plasma brain natriuretic peptide levels and left atrial appendage flow velocity in patients with non-valvular atrial fibrillation and normal left ventricular systolic function. J Echocardiogr. 2018;16(2):72-80.

34. Petersen $M$, et al. Left atrial appendage morphology is closely associated with specific echocardiographic flow pattern in patients with atrial fibrillation. Europace. 2015:17(4):539-45.

35. Boyd AC, et al. Left ventricular mass predicts left atrial appendage thrombus in persistent atrial fibrillation. Eur Heart J Cardiovasc Imaging 2013;14(3):269-75

36. January CT, et al. American College of Cardiology/American Heart Association Task Force on Practice Guidelines. 2014 AHA/ACC/HRS guideline for the management of patients with atrial fibrillation: a report of the American College of Cardiology/American Heart Association Task 
Force on Practice Guidelines and the Heart Rhythm Society. J Am Coll Cardiol. 2014;64(21):e1-76.

37. Kirchhof P, et al. ESC Scientific Document Group. 2016 ESC Guidelines for the management of atrial fibrillation developed in collaboration with EACTS. Eur Heart J. 2016;37(38):2893-962.

38. Gage BF, et al. Validation of clinical classification schemes for predicting stroke: results from the National Registry of Atrial Fibrillation. JAMA. 2001;285(22):2864-70.

\section{Publisher's Note}

Springer Nature remains neutral with regard to jurisdictional claims in published maps and institutional affiliations.
Ready to submit your research? Choose BMC and benefit from:

- fast, convenient online submission

- thorough peer review by experienced researchers in your field

- rapid publication on acceptance

- support for research data, including large and complex data types

- gold Open Access which fosters wider collaboration and increased citations

- maximum visibility for your research: over 100M website views per year

At BMC, research is always in progress.

Learn more biomedcentral.com/submissions 